\title{
BMJ Open Quality Improving the supply of critical medicines from pharmacy to reduce the delay in medicines administration on wards
}

\author{
Vitesh Patel, ${ }^{1}$ Gemma Quinn ${ }^{2}$
}

To cite: Patel V, Quinn G. Improving the supply of critical medicines from pharmacy to reduce the delay in medicines administration on wards. BMJ Open Quality 2022;11:e001417. doi:10.1136/ bmjoq-2021-001417

Received 23 March 2021 Accepted 20 December 2021

A) Check for updates

(C) Author(s) (or their employer(s)) 2022. Re-use permitted under CC BY-NC. No commercial re-use. See rights and permissions. Published by BMJ.

${ }^{1}$ Pharmacy Department, Sheffield Teaching Hospitals NHS Foundation Trust, Sheffield, UK

${ }^{2}$ School of Pharmacy and Medical Sciences, University of Bradford Faculty of Life Sciences, Bradford, UK

Correspondence to Vitesh Patel;

vitesh.patel1@nhs.net

\section{ABSTRACT}

It is nationally and locally recognised that doses of critical medications are missed or omitted on a daily basis. This has been highlighted by a National Patient Safety Agency alert published in 2010. Since then Sheffield Teaching Hospitals NHS Foundation Trusthas introduced initiatives to tackle this problem, but there are clear indications that further work is still required. The aim of this service improvement project was to improve the availability of critical medications on the ward to ensure they are available thirty minutes prior to the next scheduled dose.

Two plan-do-study-act cycles were undertaken over a 14-day period (January/February 2020) to reduce the time taken for critical medications to be supplied to the ward after a request was placed on the eOrdering system, on one care of the elderly ward. Medication request and prescription tracking data were captured during working hours each week (Monday to Sunday) and examined. The time taken for a request to be processed was captured. Following the introduction of a critical medicines checklist in the pharmacy dispensary and later a flow chart on the ward, availability of critical medication on the ward rose from $89 \%$ to $93 \%$. However, the project did not meet the project aim of ensuring $95 \%$ of critical medications requested were available on the ward.

The project highlighted that for sustainable and robust improvement, the electronic prescribing system required improvement rather than change in the work processes of the ward and pharmacy professionals.

\section{PROBLEM}

Within secondary care, missed or omitted doses of prescribed medications occur on a daily basis. The impact of this can range from no harm to death. ${ }^{1-4}$ In 2010 , The National Patient Safety Agency (NPSA) released a rapid response report asking for the immediate action of National Health Service (NHS) trusts to implement an action plan to counteract the rising number of patient safety incidents relating to missed or delayed medicines. Between September 2006 and June 2009 the NPSA received over 21000 reports of patient safety incidents relating to omitted or delayed medicines. The most serious incidents (95 cases) involved critical medications such as anti-infectives and anticoagulants. ${ }^{5}$ Since then, there have been a number of local and national initiatives to decrease the number of medicines related incidents especially related to critical medicines.

There are clear indications that further work is required to reduce the number of patient safety incidents related to missed doses of medications. Therefore this project focused on improving the supply of critical medications to ensure administration is within a timely manner.

Sheffield Teaching Hospitals NHS Foundation Trust (STHFT) is a large acute trust which operates across five different sites. It is one of three trauma centres in the region as well as providing highly specialist medical, surgical and cancer care.

In the last 12 months, the trust has implemented an electronic prescribing system (EPR) across all sites. In January 2020, there were 1325 inpatient beds which had an electronic prescription chart. The system allows the prescribing and recording of all medications administered for current admissions and previous episodes of inpatient stay. From January to December 2019, 1458436 inpatient medications were prescribed on the EPR system.

As with many NHS trusts in the UK, there is anecdotal evidence to suggest that at STHFT, there is increasing pressure on nursing staff to fit in more tasks within their working day. As a result, routine care such as administration of medications can suffer which can often lead to medications not being administered on time.

A further development has seen the introduction of a new medicines ordering system at STHFT called eOrdering. This is currently operating at three of the five sites in the trust. The system allows nurses on the ward to order medications electronically via the EPR when conducting drug rounds or on an ad-hoc basis when new medications are prescribed.

The introduction of the eOrdering system should make the medication ordering and administration process seamless. However, 
due to the combination of work pressures and the number of electronic systems at STHFT, this has stopped the benefits of the eOrdering system from being realised. This was highlighted by an audit in 2018 when the EPR system was introduced. The audit revealed that between $16 \%$ and $18 \%$ of prescribed critical medications were omitted or administered late as they were not available on the ward. ${ }^{6}$ An earlier audit in 2017, when paper drug charts were in use, found that only $10 \%$ of critical medications were being omitted or administered late. ${ }^{7}$ Despite the implementation of the eOrdering system, delays still persist. In order to improve the supply and administration of critical medications after the implementation of eOrdering, further optimisation was required to improve the process of ordering and supply of medications.

A formal benchmarking and performance monitoring process did not exist for omitted or delayed administration of critical medications at STHFT, mainly because of the difficulty in obtaining the data required across the trust. There has been a number of recent advances in the reporting process which has made it easier to extract the specific data required. The senior pharmacy management team therefore tasked to set up a project team to improve this outcome through the creation of a key performance indicator (KPI), for which the pharmacy department would be accountable to hospital management.

As a KPI did not exist for omitted or delayed critical medications, it was agreed that an appropriate target was that $95 \%$ of medications should be available on the ward within $30 \mathrm{~min}$ of the next scheduled administration. This was in line with other medication supply KPIs which also had $95 \%$ targets.

The 30-min rule as this length of time was recommended by the National Institute for Healthcare and Clinical Excellence (NICE) guidelines (QS 164) for the availability of Parkinson's medication prior to the next scheduled dose. ${ }^{8}$ This was also to be used to standardise practice across all medication groups and reduce ambiguity during the project.

The senior pharmacy management team requested that the project team consisted of key stakeholders on the ward and within the pharmacy department. Therefore, the project team consisted of the pharmacy department's clinical service manager, quality improvement (QI) pharmacist, information services manager, EPR lead pharmacist, senior ward pharmacist, ward manager, ward matron and senior sister.

The aim of the project was to ensure that $95 \%$ of critical medications, requested by nursing staff on a pilot ward, were available on the ward within thirty minutes of the next scheduled administration.

\section{BACKGROUND}

A literature search was conducted using the healthcare database advanced search. The databases searched included: AMED, EMCARE, BNI, HMIC and CINAHL using keywords and free text terms. Journal articles were excluded if they were not written in English. Within the UK, there are ongoing initiatives to move to EPR. Of the 17 articles identified, none were based on improvement in delayed doses of critical medications where an EPR is being used. This identified a gap in this area of service improvement.

The NPSA defines critical medications as medications which can result in patient death or serious harm if there are delays in their administration. Between September 2006 and June 2009, the NPSA reviewed the number of incidents reported to the national reporting and learning system relating to delayed or omitted medicines that led to severe harm or death. A total of 18527 incidents were reported, of which 27 incidents of omitted or delayed medication administration resulted in death and sixtyeight led to severe harm. Further analysis of the evidence identified nine therapeutic groups of medication of which, when administration was delayed or omitted, led to death or severe harm. The NPSA defined these nine therapeutic groups as critical medications, and recommended that NHS trusts should locally define a list of critical medications to address this risk. ${ }^{5}$

Ten per cent of incidents involving medications prescribed in secondary care have the potential to cause harm. However, $50 \%$ of them can be preventable. ${ }^{9-11}$ The NPSA published a report which identified that omitted doses was the second most common error from a data set of 10000 incidents. ${ }^{5}$ An omitted dose posed a highly significant patient safety risk to $7 \%$ of an inpatient population. $^{12}$

Further studies have shown that in non-therapeutic omission of medications the unavailability of medication is the main reason for missed doses. For every one missed dose, it increases the odds of an extra days hospital stay by $42 \%$ for all types of inpatient (non-critical care and critical care) (OR 1.42, 95\% CI 1.18 to $1.72, \mathrm{p}<0.001){ }^{13}$

A root cause analysis on dose omissions in a UK acute trust identified 54 common themes that contributed to dose omission, none of which had a greater impact than another. The co-ordination of all tasks within the process of medication supply and administration can fail at any stage. The implementation of an EPR has shown to improve administration of medicines; however introduction of these systems create new issues-such as automated dose scheduling which can again result in delays or omissions of medications.

A number of QI interventions have previously been used at NHS hospital trusts. The interventions have targeted specific parts of the medicines administration process. Toolkits have been created to categorise the risk of harm to the patient if a medication was not administered on time. A traffic light system was used to visually represent risk associated with patient harm if a dose was delayed or omitted. ${ }^{14}$ A number of NHS trusts have created standard operating procedures (SOPs) or clinical guidelines on critical medications. The documents provided guidance to ensure critical medications are given on time, and explain the procedure for the reporting of missed or 
omitted critical medications. Furthermore, each guideline or SOP outlined a specific list of critical medications, with their rationale for inclusion as a critical medication. ${ }^{15-17}$

At a national level, campaigns have been published by Parkinson's UK to highlight the importance of administering Parkinson's medications on time. The success of the campaign has resulted in the recommendations being made in national quality standard guidelines (QS 164) written by NICE. It highlights the importance of ensuring Parkinson's medications are available to administer within thirty minutes of their prescribed time to reduce the risk of adverse effects. ${ }^{818}$

\section{MEASUREMENT}

To investigate the magnitude of the problem, baseline data were collected from the EPR system and prescription tracking system. Data were retrospectively collected over a time scale of 7 days (Monday to Sunday) from one ward which already used the eOrdering system. This was completed by a single pharmacist. The pilot ward selected-a care of the elderly ward was chosen due to its lack of pharmacist and pharmacy technician cover during working hours in the week. Furthermore, the ward has a low turnover of patients, but uses multiple critical medications on a daily basis. The nursing staff were therefore highly self-sufficient to manage patients' medications on the ward and were proficient in using the EPR system.

A custom built report within the EPR system was used to identify critical medications requested from the defined critical medicines list ratified by STHFT. ${ }^{19}$ Furthermore, the report generated the date and time that a request was placed for a critical medication on eOrdering, as well as the next scheduled administration date and time, after the order was placed. The data were then cross-referenced with the prescription tracking system report that outlined the elapsed time of the clinical checking and dispensing process. The data were tabulated, and any medication requests in which the clinical checking and dispensing process were not completed before or within $30 \mathrm{~min}$ of the next scheduled administration time and date, were identified. The results were plotted on a run chart to detect any improvement and trends over the period of the QI initiative.

During the 7-day period in which the baseline data was collected, 233 medications were ordered via the eOrdering by the ward. Eighty-two requests were for critical medications, of which $11 \%$ were not available on the ward thirty minutes prior to the next scheduled administration time. Analysis of the eOrdering requests on a day-by-day basis showed 4 out of the 7 days achieved $100 \%$ compliance with the project target. On day 3, 6 and 7 which did not meet the project target; the requested critical medications included three requests for antibiotics; one for an oral anti-diabetic; three for anti-hypertensives; one for opiate pain relief and one for Parkinson's medication.

Further analysis showed that medications that were not available on the ward were administered on average
2 hours after the scheduled dose was due. However, two medication requests were placed on eOrdering only when the dose was due. The results from the baseline data collection demonstrated that improvement was required by the ward ordering critical medications in good time and a reduction in time taken to process critical medication requests within the pharmacy dispensary.

The use of the custom EPR report allowed for in-depth data collection of all requests made via the eOrdering system from the pilot ward. It also allowed for accurate analysis and measurement of the improvement made over the duration of the project. However, the disadvantage of using this method of collection was that the report was unable to gather data from the separate prescription tracking system. Each data point on the EPR report was therefore manually cross referenced with the report produced by the prescription tracking system making this method of data analysis very time consuming. It was decided by the project team that the benefits of the level detail gained from the EPR report far outweighed any disadvantages to the manual data analysis step. It was therefore, agreed that this method of data collection using manual cross referencing would be used throughout the project.

During the different phases of the project, the EPR report was setup to automatically generate a report at midnight on Sunday of each week to retrospectively collate eOrdering requests from the previous 7 days (Monday to Sunday). The report, which did not contain any patient identifiers was then sent to one member of the project team via a secure email system by 9 am on the following Monday. The data collected was stored on a secure network only accessible within STHFT through a username and password enabled computer terminal.

This method of data collection was more reliable and less intrusive on daily workflow. It also limited bias as the data collection process was automated. To ensure all critical medication orders were captured, the report was rigorously checked by the trust's information and data services department.

To ascertain if the observed outcomes were due to the QI interventions, the percentage of critical medications available on the ward within thirty minutes for each day of the 2-week period was plotted on a run chart to identify any improvements achieved.

\section{DESIGN}

Baseline data identified that a time reduction was required for a critical medication request to be processed by the pharmacy.

Due to the dynamics of the project team, most communication and updates were via email with some face-to-face meetings every 2 weeks. This strategy of communication was seen to be the most efficient method to sustain the progression of the project within the timeline agreed.

The principals from the Lean manufacturing method defined by Womack and Jones were used to try and reduce 


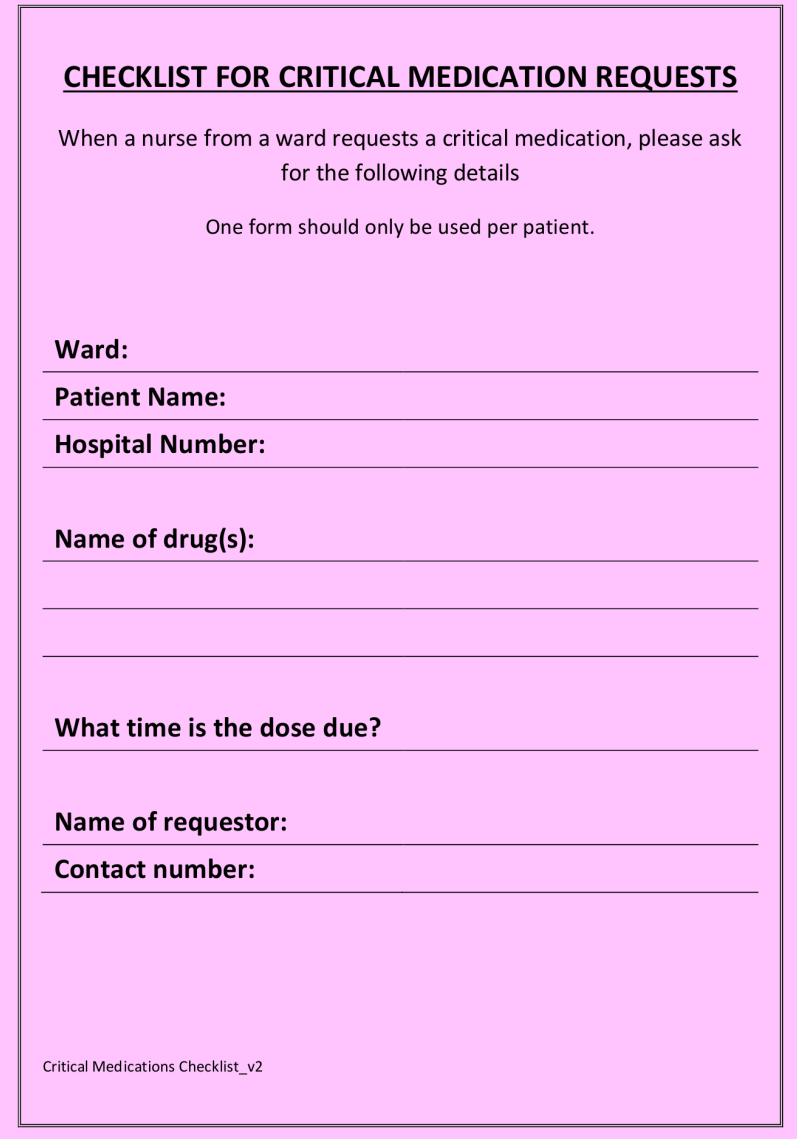

Figure 1 Critical medication checklist for requests made by nursing staff on the ward. Introduced to the pharmacy dispensary in plan-do-study-act cycle 1.

waste and create efficiency in the clinical checking and dispensing process. ${ }^{20} \mathrm{~A}$ process map was created to help visualise the journey of a prescription. At each stage in the process, data was gathered from the prescription tracking system (PTS) to identify which activity took the longest amount of time. The activity that was identified was then targeted for the QI intervention. It was identified that the clinical checking stage in the process took the longest amount of time. In order to understand the process, the clinical pharmacy standards and procedures as well as the relevant pharmacy dispensary SOPs were reviewed. The clinical standards and SOP outlined that eOrdering requests should be processed in chronological order (oldest to newest) from a central list where all requests are populated. It was also discovered that the EPR system did not have any functionality to filter requests for critical medications.

As it was not possible to improve the EPR system or change the clinical pharmacy standards/SOPs, in discussion with the project team, it was decided that a toolkit should be developed to help the nurses and checking pharmacists identify critical medication requests so that they could be prioritised. A critical medications checklist (figure 1) was created that would be completed by the dispensary staff who booked in the medication requests on the prescription tracking system so that the clinical checking pharmacists could be alerted.

In the initial stages of development, the project team met with the pharmacy dispensary manager and senior team members to try and create a checklist which did not require specialist knowledge of critical medications, but to allow easy identification of a critical medication. The dispensary staff who book in the medication requests did not have any prior knowledge of classes of medications and therefore it was important to make it as generic as possible. The initial iteration of the checklist was created and sent to the management team for review. The review prompted the checklist to be revised to reduce the number of fields required to be completed. The second iteration was sent back to the management team for review and approval. Once approved, the checklist was sent to all dispensary staff involved 2 weeks prior to the introduction of the checklist into the booking in process. This allowed time to ask questions and to gain clarity on the new procedure prior to commencement.

\section{STRATEGY}

As previously stated, the aim of the project was to ensure that $95 \%$ of critical medications requested by nursing staff on a pilot ward were available on the ward within thirty minutes of the next scheduled administration.

The data was collected over a period of fourteen days, with all eOrdering requests for critical medications recorded during working hours (Monday to Friday 9:0019:00 hours; Saturday and Sunday 9:00-16:00 hours). The project team decided to use the plan-do-study-act (PDSA) model of improvement as it allowed us to identify whether the interventions would improve the outcome on a small scale prior to a full scale roll out across the trust. Using the PDSA model allowed changes to be made easily and quickly to improve the outcome observed and report findings to key stakeholders in a short period of time.

Two PDSA test cycles were completed during the 14-day period.

\section{PDSA cycle 1}

The intervention made within the pharmacy dispensary was to introduce a checklist for the request of critical medication (figure 1). The checklist would prompt the pharmacy dispensary receptionist to ask the relevant questions to the requesting nursing staff when they telephoned the dispensary to alert them to a critical medication order. The checklist had two functions: to ensure all the information is available to the clinical pharmacist clinically verifying the request so that they can promptly complete the request; and to alert dispensing staff that there is a critical medication request which needs to be prioritised over other work. The dispensary pharmacists and technicians gave positive feedback on how it helped them prioritise their work. The intervention resulted in 
$91 \%$ of all critical medications ordered within the 7-day period to be available on the ward thirty minutes prior to administration. However, only 2 days met or exceeded the 95\% target aim.

\section{PDSA cycle 2}

It was evident during continuous monitoring and data collection over the course of the project, the ward was not ordering critical medications within a reasonable amount of time prior to the next scheduled dose. On further investigation, it was revealed that nursing staff were very confused about the process to order a critical medication through the EPR system, as well as some staff not knowing what defined a critical medication. As a result, a flow chart was created in conjunction with the ward matron and the project lead to outline the process required for ordering a critical medication through the EPR system (figure 2). Furthermore, the trust's critical medication list (figure 3) was supplied as a reminder to the nursing staff to distinguish a critical medication. With permission from the ward matron, the flow charts and critical medication lists were displayed in areas where nursing staff would access the EPR system to administer or request medications. The posters would then be referred to as required. Following the intervention, $93 \%$ of all critical medications ordered were available on the ward thirty minutes prior to

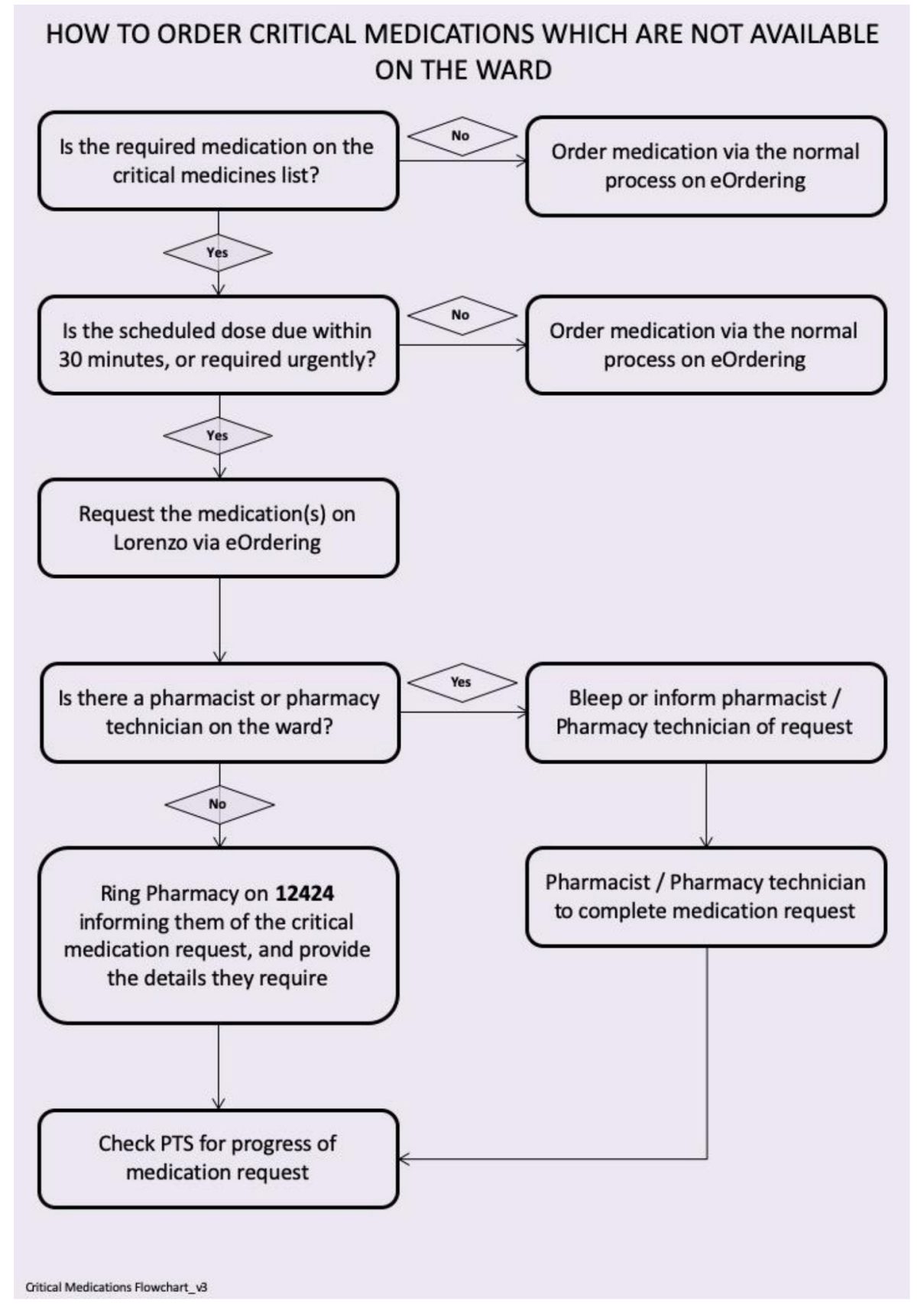

Figure 2 Flow chart for nursing staff to use when ordering critical medications on the care of the elderly ward. Introduced to the ward in plan-do-study-act cycle 2. PTS, Prescription Tracking System. 


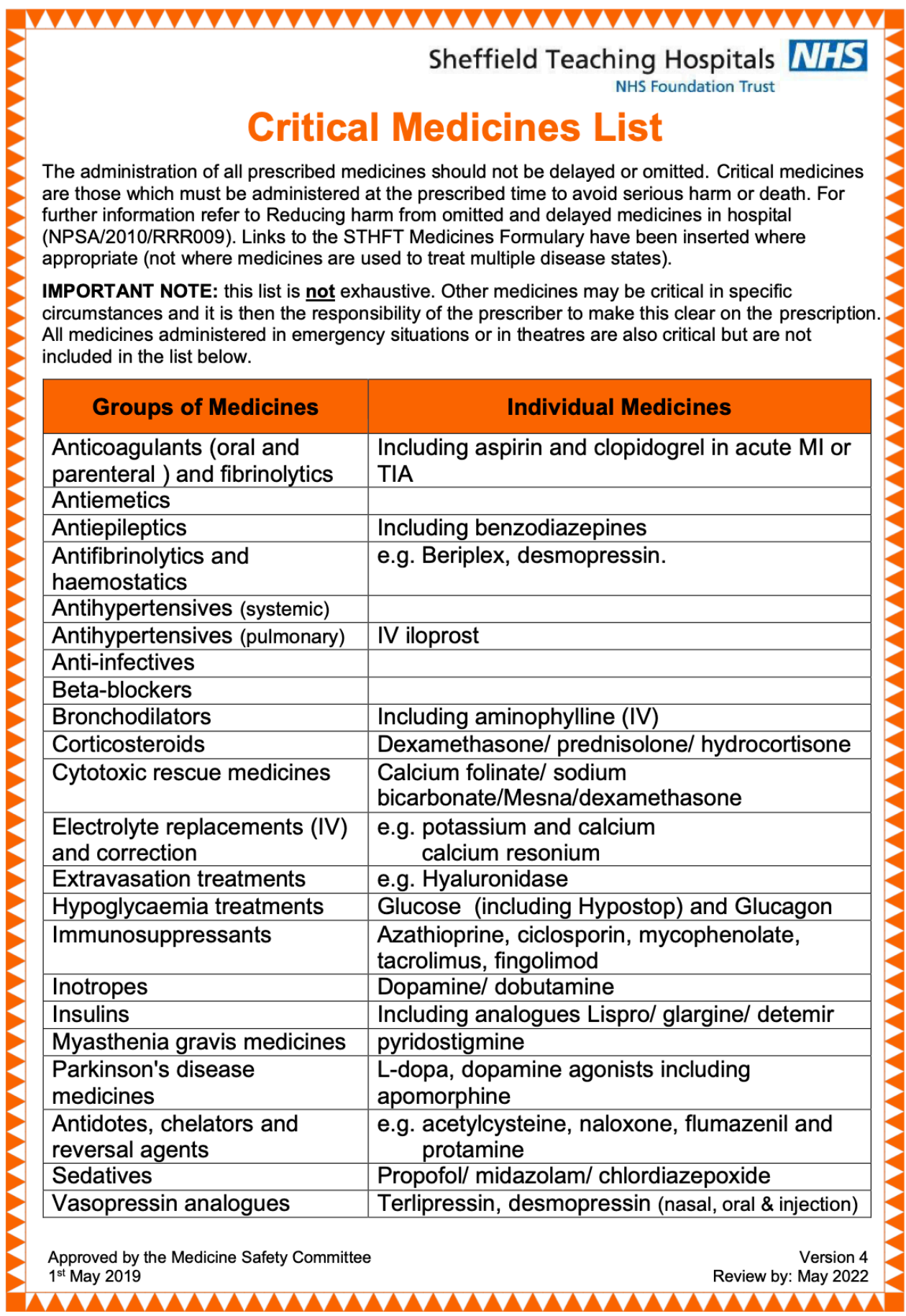

Figure 3 Critical medicines list for STHFT. MI, Myocardial Infarction; TIA, Transient Ischaemic Attack.

administration over the 7-day period but again only 2 of the 7 days met or exceeded the $95 \%$ target.

The service improvement project was conducted on the precipice of the coronavirus pandemic in 2020. As an additional piece of work, project team wanted to repeat the data collection at a later date in the future to see if the outcomes had improved or worsened. Further data collection process also took place between March 2020 and September 2020.

\section{RESULTS}

Baseline measurement of all critical medication requests via eOrdering over a 7 -day period showed that $85 \%$ of critical medications were ordered and available on the ward 30 min prior to administration time. After two PDSA cycles, an improvement was seen in the percentage of critical medications available on the ward thirty minutes prior to next scheduled dose. This increased from $89 \%$ to $93 \%$ per week (figure 4). The day of the week had no impact on the results obtained, including weekdays compared with weekends. The average time to process an ordered critical medication reduced by 1 hour and 


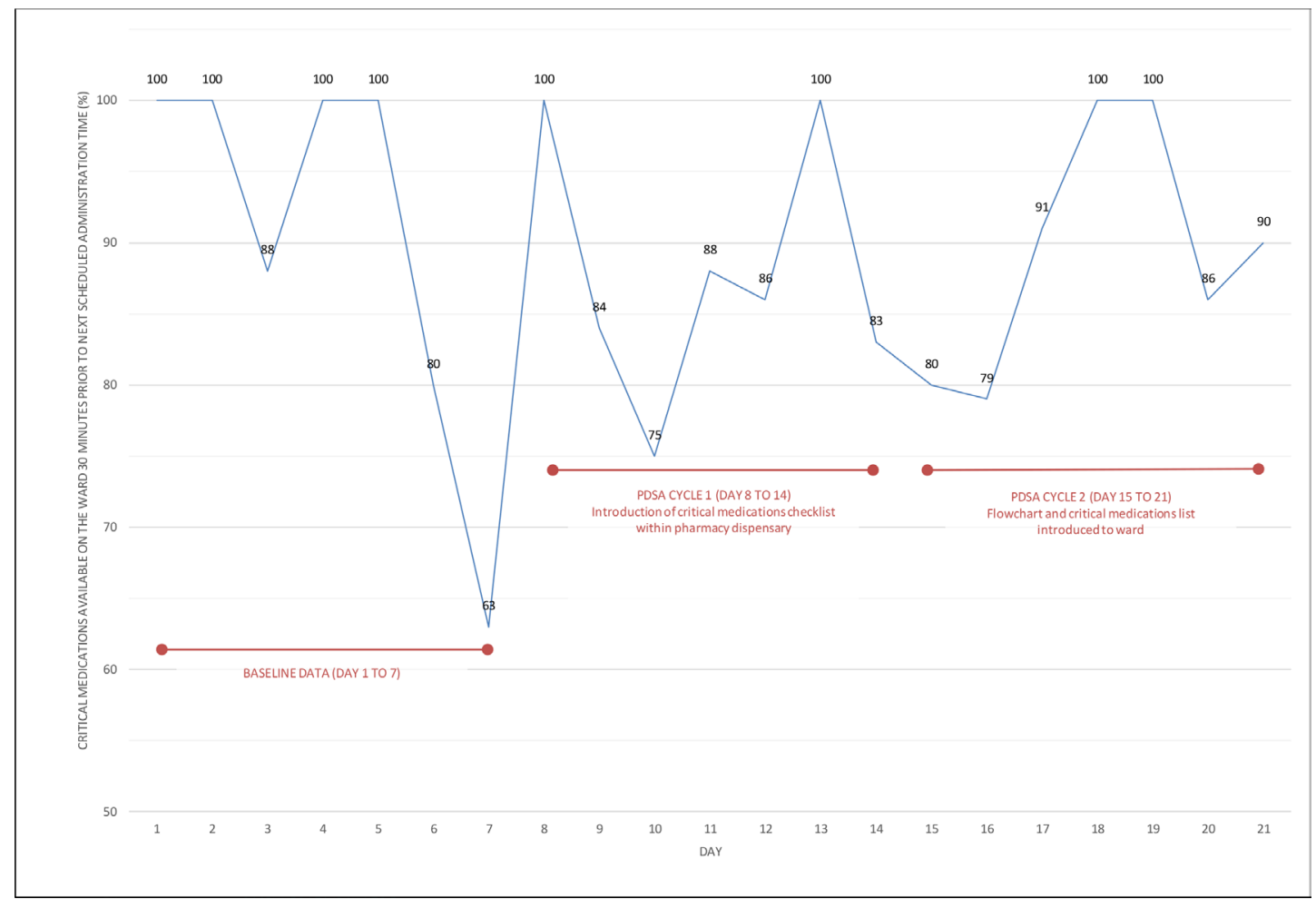

Figure 4 Run chart of the percentage of critical medications available on the ward on a day-by-day basis throughout the project. PDSA, plan-do-study-act.

$14 \mathrm{~min}$, and the average time a critical medication was ordered by the ward prior to the next scheduled dose by the ward improved by 3 hours and $45 \mathrm{~min}$. Due to the nature of the data collection process, the data used were deemed complete and accurate.

The results from the data collection which took place between March 2020 and September 2020, showed that only $62 \%$ of critical medications were available on the ward $30 \mathrm{~min}$ prior to the next scheduled dose. The average time to process a critical medication increased by $52 \mathrm{~min}$, in comparison to the results obtained during PDSA cycle 2. However, the average time between ordering a critical medication by the ward and the next schedule dose improved by 1 hour and $9 \mathrm{~min}$.

\section{LESSONS AND LIMITATIONS}

The aim of the project was to improve the availability of critical medications on the ward after they have been requested through the eOrdering system. This was to ensure they are available on the ward $30 \mathrm{~min}$ before the next scheduled dose was due. Changes were made on one pilot ward, with the intention to implement sustainable changes across STHFT. The improvement was prompted by previous clinical audits which highlighted that action was required to improve the number missed doses of critical medications. Furthermore, baseline data gathered from the EPR system suggested that it was an on-going issue following the initial audits in 2017 and 2018.

Key lessons gained from this project were the importance of identifying key stakeholders at the early stages in the project, which can ensure that the progression of the project and implementation at ward and directorate level can be achieved. ${ }^{21}$ Furthermore, the project highlighted that open, honest and regular communication is key to ensure that all individuals involved in any part of the system are aware of changes made, resulting in increased compliance.

The project strengths were gained from the ability to gather data from the EPR system without hindering workflow on the wards and within the pharmacy dispensary in real-time. The ability to have automated data collection was a major time saving aspect to the project, allowing more time for other tasks. It also enabled the project lead to be more readily available to action any outstanding tasks.

As improvement in the supply of critical medications lay at the core of this project, we did not anticipate that the introduction of a new system would create further complications to the clinical checking and dispensing process within the pharmacy. At present, the dispensary pharmacist has to simultaneously manage five different work streams. Adding another process led the dispensary pharmacists to experience undue stress which resulted in cognitive and system fatigue. Matthews and Hancock ${ }^{22}$ describe that fatigue and stress are interconnected, and can be derived from three different loci: input, adaptation and output. In the case of introducing a new system, it could be argued that an additional input stressor increased the level of stress and pressure on the pharmacist, reducing their productivity. 
The project also highlighted that there was a barrier to ensuring all pharmacists and technicians prioritise the work in the same way. Many members of staff within the dispensary were accustomed to their way of working and therefore may have required a longer length of time to embrace a novel way of processing work. This led to different pharmacists and technicians making different decisions on what work should be prioritised over another, which could have influenced the outcome of the results.

The project's limitations may have influenced the outcomes observed in the results. As the project was only conducted on one ward, and for a 14-day period, the sample size (requests on eOrdering) was small. Data collection over a longer period of time would have been preferable.

Although the project did not meet the specified target, it was met with acceptance from all stakeholders that were involved who were very keen to make the project work. But to make it sustainable to implement across the trust, future improvements would be required. If the EPR system could alert a user to a critical medication that has been ordered, it would remove the decision making step on how work is prioritised. This would reduce variability between different dispensary pharmacists and technicians, and remove the need to implement policy change.

The additional data collection highlighted that the coronavirus pandemic had heavily affected routine procedures on the ward. This was most likely due to healthcare staff shortages across both the nursing and pharmacy teams. Furthermore, this period of time, many members of healthcare staff were redeployed to help with the efforts to treat those with coronavirus on other wards in the trust. This would have likely to have had a negative effect on the level of care to those patients who were on the care of the elderly ward. This was due to using nursing staff that were unfamiliar with the standard procedures on the ward.

\section{CONCLUSION}

It is nationally recognised that the delay in the administration of critical medications can lead to patient harm. It is locally recognised at STHFT that since the inception of the EPR system, delays in administration of critical medications still persist. There has been limited research conducted in this area of QI, however many NHS trusts in the UK have implemented their own methods of tackling this problem.

Our aim was to ensure $95 \%$ of all critical medications requested were available on the ward within thirty minutes of the next scheduled dose after a series of QI interventions. Although we were unable to meet the specific aim of the project, there was a moderate improvement in the number of critical medications available on the ward $(89 \%-93 \%)$.

During the progression of the project, the aim and objectives were not changed, but our focus on tackling the problem became more holistic when the issue of the ward not ordering medication in a timely manner was uncovered. Due to the limitations on the timescale of data collection sample size, a larger project would be required to implement a sustainable change throughout the trust. To overcome any variability or limitations to the project it would be ideal to improve systems to aid with the clinical decision-making processes.

Acknowledgements The author would like to thank the EPMA and information services team, all the staff within the pharmacy department, and on the care of the elderly ward at STHFT who supported this project.

Contributors VP have contributed $70 \%$ to the planning, conduct, data collection and reporting of results described in this article. GQ has contributed $30 \%$ towards the planning, research methodology and reporting of results described in this article. VP accepts full responsibility as guarantor for the work carried out in this service improvement project

Funding The authors have not declared a specific grant for this research from any funding agency in the public, commercial or not-for-profit sectors.

Competing interests None declared.

Patient and public involvement Patients and/or the public were not involved in the design, or conduct, or reporting, or dissemination plans of this research.

Patient consent for publication Not required.

Ethics approval The project did not require ethical approval at STHFT, as it was service improvement. Ethical approval was granted by the University of Bradford Biomedical, natural, physical and health sciences research ethics panel (ref: EC25924).

Provenance and peer review Not commissioned; externally peer reviewed.

Data availability statement All data relevant to the study are included in the article.

Open access This is an open access article distributed in accordance with the Creative Commons Attribution Non Commercial (CC BY-NC 4.0) license, which permits others to distribute, remix, adapt, build upon this work non-commercially, and license their derivative works on different terms, provided the original work is properly cited, appropriate credit is given, any changes made indicated, and the use is non-commercial. See: http://creativecommons.org/licenses/by-nc/4.0/.

\section{REFERENCES}

1 Coleman JJ, McDowell SE, Ferner RE. Dose omissions in hospitalized patients in a UK Hospital: an analysis of the relative contribution of adverse drug reactions. Drug Saf 2012;35:677-83.

2 Coleman JJ, Hodson J, Brooks HL, et al. Missed medication doses in hospitalised patients: a descriptive account of quality improvement measures and time series analysis. Int J Qual Health Care 2013;25:564-72.

3 Morley C, McLeod E, McKenzie D, et al. Reducing dose omission of prescribed medications in the hospital setting: a narrative review. Drugs Ther Perspect 2016;32:203-8.

4 Warne S, Endacott R, Ryan H, Chamberlain W, et al. Non-therapeutic omission of medications in acutely ill patients. Nurs Crit Care 2010;15:112-7.

5 National Patient Safety Agency. Rapid response report NPSA/2010/ RRR009: reducing harm form omitted and delayed medicines in hospital. London: National patient safety agency, 2010.

6 Ming Cho K. Re-audit of omitted doses of regular medicines prescribed on inpatient drug charts. Sheffield: STHFT, 2018.

7 Whitaker R, Dorward B, Thomas N. To determine the reasons for 'drug not avaiable' which result in drug dosage omissions of regular medications on the neurology wards. Sheffield: STHFT, 2017.

8 National institute for healthcare and clinical excellence (NICE). Parkinson's disease: quality standard (QS 164). NICE. Available: https://www.nice.org.uk/guidance/qs164 [Accessed 1 Nov 2019].

9 National patient safety agency Safety in doses: medications safety incidents in the NHS. The fourth report from the patient safety observatory (PSO/4). London: National patient safety agency, 2007.

10 Baqir W, Jones K, Horsley W, et al. Reducing unacceptable missed doses: pharmacy assistant-supported medicine administration. Int J Pharm Pract 2015;23:327-32. 
11 Vincent C, Neale G, Woloshynowych M. Adverse events in British hospitals: preliminary retrospective record review. BMJ 2001;322:517-9.

12 Green CJ, Du-Pre P, Elahi N, et al. Omission after admission: failure in prescribed medications being given to inpatients. Clin Med 2009;9:515-8.

13 Warne S, Endacott R, Ryan H, Chamberlain W, et al. Non-therapeutic omission of medications in acutely ill patients. Nurs Crit Care 2010;15:112-7.

14 Barrett T, Beswick T, Bischler A, et al. NPSA rapid response report: reducing harm from omitted and delayed medicines in hospital. $A$ tool to support local implementation. UK medicines information. Available: https://www.sps.nhs.uk/articles/npsa-rapid-responsereport-reducing-harm-from-omitted-and-delayed-medicines-inhospital-a-tool-to-support-local-implementation/ [Accessed 20 Oct 2019].

15 Jenkinson M. Continuity of critical medicines - clinical guidelines. East Kent hospitals university NHS Foundation trust. Available: https://www.sps.nhs.uk/wp-content/uploads/2016/08/F-EKHUFT_ Continuity_of_Critical_Medicines_Clinical_Guideline_1.1.pdf [Accessed 10 Oct 2019].

16 Morgan L. N. SOP: reducing harm from omitted medicines and delayed medicines in bedded services and Hmp wormwood scrubs. central London community healthcare NHS trust. Available: https://www.sps.
nhs.uk/wp-content/uploads/2016/08/G-CLCH_Omitted_and_Delayed Final Version_2-September 2013.pdf [Accessed 10 Oct 2019].

17 Oborne A. Clinical guideline - omitted or delayed doses: critical drugs list. Guy's and St Thomas' NHS foundation trust. Available: https://www.sps.nhs.uk/wp-content/uploads/2016/08/I-GSTT_ Omitted_or_delayed_doses_Critical_drugs_list_v3.pdf [Accessed 10 Oct 2019].

18 Parkinson's UK. Get it on time - the case for improving medication management for Parkinson's. Available: https://www.parkinsons. org.uk/sites/default/files/2019-10/CS3380\%20Get\%20it\%20on\% 20Time\%20Report\%20A4\%20final\%2026.09.2019-compressed\% 20\%281\%29.pdf [Accessed 1 Feb 2020].

19 Sheffield Teaching Hospitals NHS Foundation Trust. Critical medicines list. Sheffield: STHFT, 2019.

20 Womack JP, Jones DT. Lean thinking: banish waste and create wealth in your organisation. New York: Simon and Schuster, 1996.

21 ACT Academy. Online library of quality service improvement and redesign tools - stakeholder analysis. Available: https://improvement. nhs.uk/documents/2169/stakeholder-analysis.pdf [Accessed 1 Feb 2020].

22 Matthews G, Hancock PA. The Handbook of operator fatigue. Farnham: CRC Press LLC, 2012. 